\title{
Prevalence of thyroid dysfunction in patients with COVID-19: a systematic review
}

\author{
Luca Giovanella $^{1,2}$ (1) $\cdot$ Rosaria M. Ruggeri ${ }^{3}$ Petra Petranović Ovčariček ${ }^{4}$. Alfredo Campenni ${ }^{5}$. Giorgio Treglia ${ }^{1,6,7,8}$. \\ Desiree Deandreis ${ }^{9}$
}

Received: 17 January 2021 / Accepted: 25 February 2021 / Published online: 11 March 2021

(C) Italian Association of Nuclear Medicine and Molecular Imaging 2021

\begin{abstract}
Purpose Currently, there is an increasing interest regarding the impact of COVID-19 on the thyroid function. As several recent reports have described the onset of thyroid dysfunction in patients diagnosed with COVID-19, we performed a systematic review to assess the prevalence of thyroid dysfunction in patients with COVID-19 as this information could be clinically relevant for the management of these patients.

Methods A comprehensive computer literature search using PubMed/Medline and Cochrane databases was performed until November 14, 2020 to search original articles evaluating thyroid dysfunction in COVID-19 patients. Information about thyroid dysfunction assessed by thyroid function test was retrieved by the eligible articles. Qualitative analysis (systematic review) only was performed whether a significant heterogeneity of data was detected.

Results Seven studies including 1237 patients with COVID-19 were included. A significant heterogeneity across the studies was found. Most COVID-19 patients were euthyroid with TSH levels in the normal range (from 44 to $94 \%$ of the COVID-19 patients assessed in the included studies). The prevalence of thyroid dysfunction in COVID-19 patients (defined as abnormal thyroid function tests) largely varies among the included studies between 13 and 64\% of COVID-19 patients as well as clinical presentation. A positive correlation between thyroid dysfunction and clinical severity of COVID-19 was reported. Conclusion Literature data show that thyroid dysfunction is present in a significant percentage of patients with COVID-19. Assessment of thyroid function may be considered in symptomatic COVID-19 patients to have a baseline before introducing thyroid-interfering drugs and those requiring high-intensity care. Further, well-designed studies are needed to better elucidate the impact of COVID-19 on thyroid function and inform thyroid function testing and thyroid dysfunction management in COVID-19 patients.
\end{abstract}

Keywords COVID-19 $\cdot$ SARS-CoV-2 $\cdot$ Thyroid $\cdot$ TSH $\cdot$ Thyroid dysfunction $\cdot$ Systematic review

Luca Giovanella

luca.giovanella@eoc.ch

1 Clinic for Nuclear Medicine and Competence Centre for Thyroid Diseases, Imaging Institute of Southern Switzerland, Ente Ospedaliero Cantonale, Via A. Gallino 12, 6500 Bellinzona, Switzerland

2 Clinic for Nuclear Medicine, University Hospital and University of Zurich, Zürich, Switzerland

3 Unit of Endocrinology, Department of Clinical and Experimental Medicine, University of Messina, Messina, Italy

4 Department of Oncology and Nuclear Medicine, University Hospital Center "Sestre Milosrdnice", Zagreb, Croatia
5 Unit of Nuclear Medicine, Department of Biomedical and Dental Sciences and Morpho-Functional Imaging, University of Messina, Messina, Italy

6 Faculty of Biology and Medicine, University of Lausanne, Lausanne, Switzerland

7 Academic Education, Research and Innovation Area, Ente Ospedaliero Cantonale, Bellinzona, Switzerland

8 Faculty of Biomedical Sciences, Università della Svizzera italiana, Lugano, Switzerland

9 Nuclear Medicine Division, Department of Medical Sciences, AOU Città della Salute e della Scienza, University of Turin, Turin, Italy 


\section{Introduction}

COronaVIrus Disease (COVID-19) is an infectious disease caused by the SARS-CoV-2 (Severe Acute Respiratory Syndrome CoronaVirus 2) which is able to enter the human cells through the angiotensin-converting enzyme 2 (ACE2) receptor [1]. COVID-19 mainly affects the lower respiratory tract and causes flu-like symptoms. In severe cases, COVID-19 may cause interstitial pneumonia that may evolve in acute respiratory distress syndrome and death [1]. However, it has been recently demonstrated that COVID-19 may have widespread effects throughout the body with several clinical manifestations including the endocrine organs which can be impaired by different mechanisms, such as direct virus damage to the gland or to an indirect effect on the hypothalamus-pituitary gland axis, systemic inflammation due to the secretion of cytokines and chemokines, vascular derangement and autoimmune reactions [2,3]. Several recent reports have described the onset of thyroid dysfunction in previously thyroid healthy patients diagnosed with COVID-19 (from subclinical thyroid hormone derangement to overt thyrotoxicosis due to subacute thyroiditis) as well as the potential negative impact of COVID-19 on patients with pre-existing thyroid diseases. In addition, ACE-2 receptors expression was demonstrated in thyroid follicular cells, making them a potential target for SARS-CoV-2 entry [4] (Fig. 1). Our aim is to summarize available literature on the field and perform a systematic review to assess the prevalence of thyroid dysfunction in patients with COVID19 as this information could be clinically relevant for the management of these patients.

\section{Materials and methods}

\section{Search strategy}

Four authors (LG, PPO, GT, DD) performed a comprehensive computer literature search of PubMed/MEDLINE and Cochrane library databases according to the PRISMA statement [5] to find published articles investigating the prevalence of thyroid dysfunction in patients with COVID19. We have defined thyroid dysfunction as abnormal thyroid function tests (TSH and/or thyroid hormones). A search algorithm was created using a combination of these terms: (A) "COVID" OR "SARS" AND (B) "thyroid". The search was performed and updated until November 14, 2020. No language or time restrictions were applied. To expand the search, references of the retrieved articles were also screened for potential additional studies.

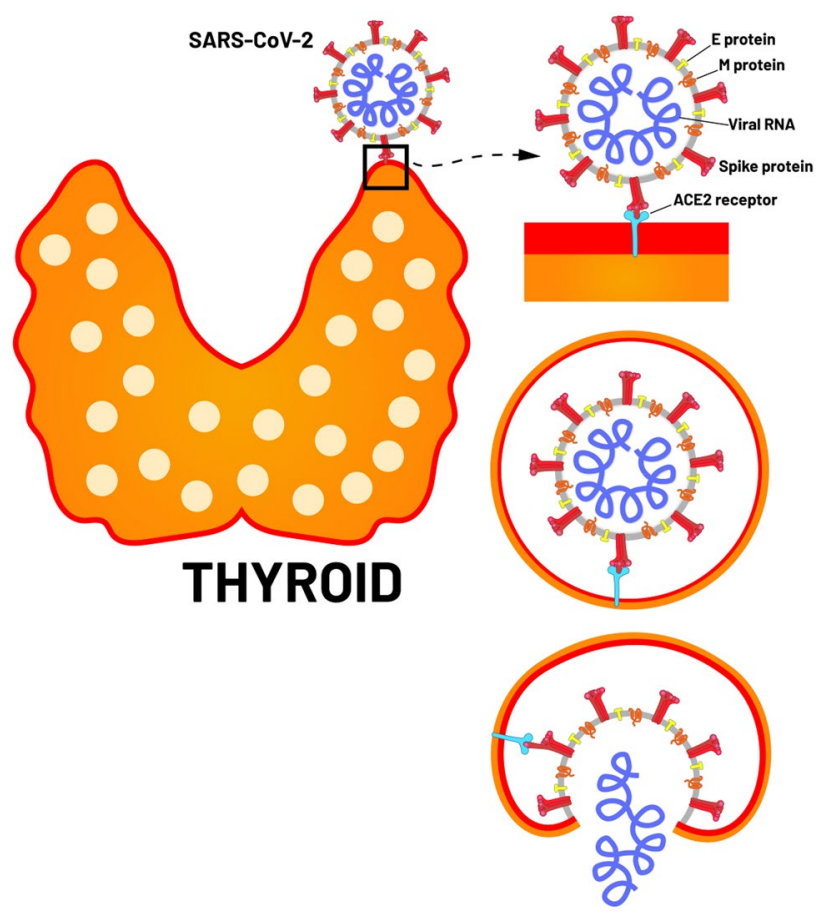

Fig. 1 ACE-2 receptors in thyroid follicular cells and SARS-CoV-2 entry

\section{Study selection}

Studies investigating the prevalence of thyroid dysfunction defined as abnormal thyroid function tests (including thyrotropin and/or thyroid hormones) in COVID-19 patients without pre-existing thyroid diseases were eligible for inclusion in this review. The exclusion criteria were: (a) articles not within the field of interest of this review (including articles with COVID-19 patients and pre-existing thyroid diseases); (b) review articles, editorials or letters, comments, conference proceedings; (c) case reports and small case series. Four reviewers (LG, PPO, GT, DD) independently reviewed the titles and abstracts of the retrieved articles, applying the inclusion and exclusion criteria mentioned above. Articles were rejected if they were clearly ineligible. Four researchers then independently reviewed the full-text version of the selected articles to assess their eligibility for inclusion. Disagreements were resolved through an online consensus meeting.

\section{Data extraction}

For each eligible study, information was collected independently by four reviewers about basic study (authors, year of publication, study design), patient characteristics 
(number of COVID-19 patients assessed, mean age and sex ratio), and main findings including prevalence of abnormal thyroid function tests and in particular prevalence of abnormal thyrotropin (TSH) values in COVID-19 patients.

\section{Quality assessment}

The overall quality of the studies included in this systematic review was appraised based on the National Heart, Lung and Blood Institute Study Quality Assessment Tools [https://www.nhlbi.nih.gov/health-topics/study-qualityassessment-tools (accessed on November 14, 2020)].

\section{Data analysis}

The calculation of a pooled prevalence of thyroid dysfunction in COVID-19 patients was planned only whether a significant heterogeneity among the studies was not detected. In case of detection of significant heterogeneity among the studies, a qualitative analysis (systematic review) without quantitative analysis (meta-analysis) was carried out.

\section{Results}

\section{Literature search}

A summary of the literature search process is showed in Fig. 2. The comprehensive computer literature search from

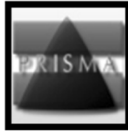

Research question: Thyroid dysfunction in patients with COVID-19

Research string: ((COVID) OR (SARS)) AND (thyroid)

Database screened: PubMed /MEDLINE and Cochrane library until November $14^{\text {th }} 2020$

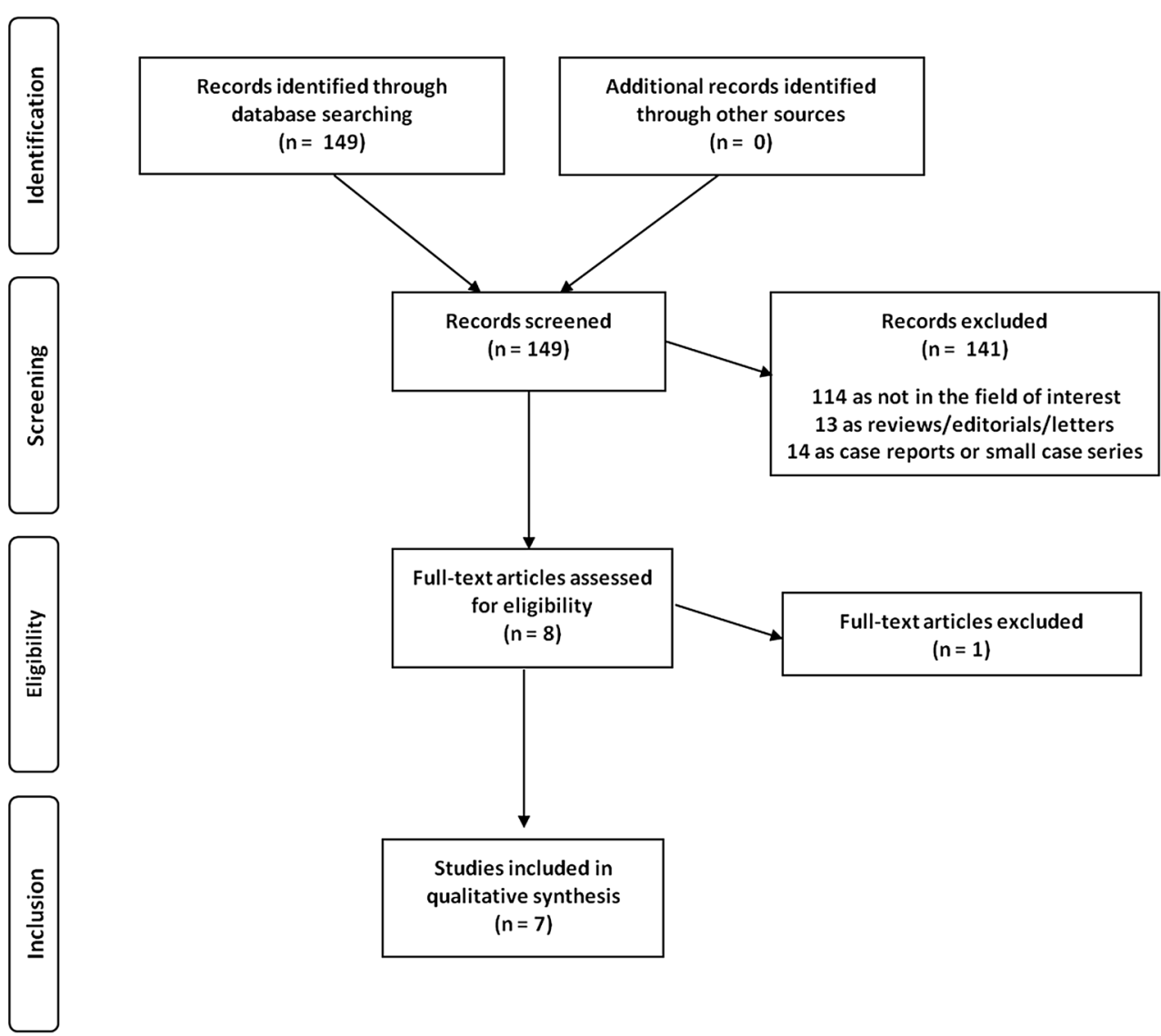

Fig. 2 Flow chart of the search for eligible studies on thyroid dysfunction in COVID-19 patients 
PubMed/MEDLINE and Cochrane library database revealed 149 records. Among these records, 141 were excluded (114 because outside the field of interest, 13 as reviews/editorials/letters, 14 as case reports or small case series). Eight original articles were selected and retrieved in full-text version [6-13]. One article was excluded after reading the full text [6]. Further additional studies screening the references of the selected articles were not found. Therefore, 7 studies including 1237 patients with COVID-19 were eligible for the qualitative synthesis (systematic review) [7-13].

\section{Qualitative analysis (systematic review)}

Main characteristics of the included studies are summarized in Tables 1 and 2 . Seven recently published studies by researchers from China ( 4 articles), Italy ( 2 articles) and United Kingdom (1 article) evaluated thyroid dysfunction in patients with COVID-19 [7-13]. Both prospective $(n=3)$ and retrospective $(n=4)$ studies were included. All the studies were single-center studies and the study quality was judged as fair according to the selected quality assessment tool. COVID-19 patients included in the eligible articles showed different characteristics; in particular, COVID19 patients with different clinical disease severity were included (Table 1). As showed by Tables 1 and 2, a significant heterogeneity across the studies was found. Therefore, a meta-analysis on the prevalence of thyroid dysfunction in COVID-19 patients was not performed and main findings are briefly summarized here below through a qualitative synthesis. About the prevalence of thyroid dysfunction in COVID-19 patients, defined as abnormal thyroid function tests (TSH and/or thyroid hormones), it largely varies among the included studies between 13 and 64\% of COVID-19 patients. In particular, both cases of thyrotoxicosis, related mainly to thyroiditis and more rarely to the new onset of Grave' disease, and hypothyroidism were reported. Low TSH levels were reported in 5\% up to $54 \%$ of patients with COVID-19, whereas high TSH levels were reported in $0 \%$ up to $8 \%$ of patients with COVID-19 [7-13]. In three studies the prevalence of thyroid dysfunction found in COVID-19 patients was significantly higher compared to a control group of patients (including healthy controls and non-COVID-19 patients), in particular, the mean TSH values were significantly lower in COVID-19 patients compared to control groups $[7,9,11]$. Furthermore, a particular condition, the so-called euthyroid sick syndrome (ESS), characterized by low free-T3 (FT3) and, less frequently, low free-T4 (FT4) in combination with normal or low TSH has been described in severely ill patients [13].

In particular, four studies reported a correlation between thyroid dysfunction and clinical severity of COVID-19, in particular, the degree of the decrease in TSH levels was positively correlated with the severity of the disease $[7,8,11$, 13]. About the correlation between thyroid dysfunction and mortality in patients with COVID-19, controversial findings were reported in the included studies when this correlation was assessed $[8,10,13]$. One study reported that reduced FT3 independently predicted all-cause mortality of patients

Table 1 Basic study and patients characteristics

\begin{tabular}{|c|c|c|c|c|c|c|c|}
\hline Authors & Year & Country & Study design & Study quality* & COVID-19 patients & $\begin{array}{l}\text { Age of COVID-19 } \\
\text { patients (years) }\end{array}$ & $\begin{array}{l}\text { Male percentage in } \\
\text { COVID-19 patients }\end{array}$ \\
\hline Chen et al. & 2020 & China & $\begin{array}{l}\text { Retrospective single } \\
\text { center }\end{array}$ & Fair & $\begin{array}{l}\text { Overall: } 50 \\
\text { non-severe: } 15(30 \%) \\
\text { severe: } 23(46 \%) \\
\text { critical: } 12(24 \%)\end{array}$ & NR & NR \\
\hline Gao et al. & 2020 & China & $\begin{array}{l}\text { Retrospective single } \\
\text { center }\end{array}$ & Fair & $\begin{array}{l}\text { Overall: } 100 \\
\text { non-severe: } 34(34 \%) \\
\text { severe/critical: } 66 \\
\quad(66 \%)\end{array}$ & Mean: 62 & $52 \%$ \\
\hline Khoo et al. & 2020 & UK & $\begin{array}{l}\text { Prospective single } \\
\text { center }\end{array}$ & Fair & Overall: 334 & Mean: 66 & $61 \%$ \\
\hline Lania et al. & 2020 & Italy & $\begin{array}{l}\text { Retrospective single } \\
\text { center }\end{array}$ & Fair & Overall: 287 & Median: 66 & $67 \%$ \\
\hline Lui et al. & 2020 & China & $\begin{array}{l}\text { Prospective single } \\
\text { center }\end{array}$ & Fair & $\begin{array}{l}\text { Overall: } 191 \\
\text { non-severe: } 185(97 \%) \\
\text { severe. } 6(3 \%)\end{array}$ & Mean: 53.5 & $52 \%$ \\
\hline Muller et al. & 2020 & Italy & $\begin{array}{l}\text { Prospective single } \\
\text { center }\end{array}$ & Fair & $\begin{array}{l}\text { Overall: } 126 \\
\text { non-severe: } 41(33 \%) \\
\text { severe: } 85(67 \%)\end{array}$ & $\begin{array}{l}\text { Mean: } 65 \text { and } 70 \text { in two } \\
\text { groups }\end{array}$ & $61 \%$ \\
\hline Zou et al. & 2020 & China & $\begin{array}{l}\text { Retrospective single } \\
\text { center }\end{array}$ & Fair & Overall: 149 & Median: 47 & $48 \%$ \\
\hline
\end{tabular}

$*$ = according to the National Heart, Lung and Blood Institute quality assessment tools; $N R$ not reported 
Table 2 Main outcomes of the included studies

\begin{tabular}{|c|c|c|c|c|c|c|c|c|c|}
\hline Authors & $\begin{array}{l}\text { Abnormal } \\
\text { TFT }\end{array}$ & $\begin{array}{l}\text { Low TSH } \\
\text { levels }\end{array}$ & $\begin{array}{l}\text { Normal } \\
\text { TSH level }\end{array}$ & $\begin{array}{l}\text { High TSH } \\
\text { levels }\end{array}$ & TT3-TT4 & fT3-fT4 & $\begin{array}{l}\text { Differences } \\
\text { of TFT } \\
\text { (COVID-19 } \\
\text { vs controls) }\end{array}$ & $\begin{array}{l}\text { Correlation } \\
\text { of TFT vs } \\
\text { COVID-19 } \\
\text { severity }\end{array}$ & $\begin{array}{l}\text { Correlation of } \\
\text { thyroid dys- } \\
\text { function with } \\
\text { COVID-19 } \\
\text { mortality }\end{array}$ \\
\hline Chen et al. & $32 / 50(64 \%)$ & $\begin{array}{l}28 / 50^{1} \\
\quad(56 \%)\end{array}$ & $\begin{array}{c}32 / 50^{1} \\
(44 \%)\end{array}$ & $0 / 50^{1}(0 \%)$ & $\begin{array}{c}\text { Low TT3 } \\
15 / 50 \\
(30 \%)^{7} \\
\text { Low TT4 } \\
3 / 50 \\
(6 \%)^{7}\end{array}$ & NR & Yes & Yes & NR \\
\hline Gao et al. & NR & $7 / 100^{2}(7 \%)$ & $\begin{array}{r}85 / 100^{2} \\
(85 \%)\end{array}$ & $8 / 100^{2}(8 \%)$ & - & $\begin{array}{l}\text { Low fT3 } \\
28 / 100 \\
(28 \%)^{8} \\
\text { High fT3: } \\
\text { none } \\
\text { Low fT4 } 4^{8} \\
\text { 3/100 (3\%) } \\
\text { High fT4 } 4^{8} \\
17 / 100 \\
(17 \%)\end{array}$ & NR & Yes & Yes \\
\hline Khoo et al. & $\begin{array}{l}45 / 334 \\
\quad(13.5 \%)\end{array}$ & $\begin{array}{r}18 / 334^{3} \\
(5.4 \%)\end{array}$ & $\begin{array}{r}297 / 334^{3} \\
(88.9 \%)\end{array}$ & $\begin{array}{r}19 / 334^{3} \\
(5.7 \%)\end{array}$ & - & $\begin{array}{l}\text { fT3 not } \\
\text { reported } \\
\text { High fT4 } \\
\text { none }{ }^{9} \\
\text { Low fT4 } \\
{ }^{9} 10 / 289 \\
(3.4 \%)\end{array}$ & Yes & NR & NR \\
\hline Lania et al. & $\begin{array}{l}73 / 287 \\
\quad(25.4 \%)\end{array}$ & $\begin{array}{l}58 / 287^{4} \\
(20.2 \%)\end{array}$ & $\begin{array}{r}214 / 287^{4} \\
(74.6 \%)\end{array}$ & $\begin{array}{r}15 / 287^{4} \\
(5.2 \%)\end{array}$ & - & $\begin{array}{l}\text { High fT3- } \\
\text { fT4 } 31 / 73 \\
(42 \%)^{10} \\
\text { Low fT3 and } \\
\text { fT4 } 2 / 73 \\
(2.8 \%)^{10}\end{array}$ & NR & NR & Yes \\
\hline Lui et al. & $\begin{array}{l}25 / 191 \\
\quad(13.1 \%)\end{array}$ & $\begin{array}{r}11 / 191^{5} \\
(5.8 \%)\end{array}$ & $\begin{array}{r}179 / 191^{5} \\
(93.7 \%)\end{array}$ & $\begin{array}{l}1 / 191^{5} \\
(0.5 \%)\end{array}$ & - & $\begin{array}{l}\text { Low fT3 } \\
\text { levels } \\
12 / 191 \\
(6.7 \%)^{12} \\
\text { Borderline- } \\
\text { raised } \\
\text { fT4 3/191 } \\
(1.6 \%)^{12}\end{array}$ & NR & Yes & NR \\
\hline Muller et al. & $\begin{array}{l}32 / 126 \\
(25.4 \%)\end{array}$ & $\begin{array}{l}25 / 126^{6} \\
(19.8 \%)\end{array}$ & $\begin{array}{l}94 / 126^{6} \\
(74.6 \%)\end{array}$ & $\begin{array}{l}7 / 126^{6} \\
(5.6 \%)\end{array}$ & - & $\begin{array}{l}\text { Higher fT4 } \\
\text { levels vs } \\
\text { controls }^{11} \\
\text { No differ- } \\
\text { ences } \\
\text { in fT3 } \\
\text { levels }^{11}\end{array}$ & Yes & No & NR \\
\hline Zou et al. & $\begin{array}{l}41 / 149 \\
(27.5 \%)\end{array}$ & NR & NR & NR & - & $\begin{array}{l}\text { Low fT3 } \\
\quad 41 / 149 \\
(27.5 \%)^{13}\end{array}$ & NR & Yes & No \\
\hline
\end{tabular}

NA not available; Reference range TSH: ${ }^{1} \mathrm{Abbott}$ (not reported), ${ }^{2}$ Roche $(0.27-4.20 \mathrm{mUI} / \mathrm{L}),{ }^{3} \mathrm{Abbott}(0.3-4.20 \mathrm{mUI} / \mathrm{L}),{ }^{4} \mathrm{Beckman}(0.34-4.80$ $\mathrm{mUI}(\mathrm{L}),{ }^{5}$ Cobas, ROCHE (0.27-4.2 mUI/L), ${ }^{6}$ ADVIA CentaurTSH3-Ultra (0.35-4.8 mIU/L); Reference Range Thyroid Hormones: ${ }^{7}$ Not reported, ${ }^{8} \mathrm{fT} 3=3.10-6.8 \mathrm{pmol} / \mathrm{L}$ fT4 $=12-22 \mathrm{pmol} / \mathrm{L},{ }^{9} \mathrm{fT} 4=9.0-23 \mathrm{pmol} / \mathrm{L},{ }^{10} \mathrm{fT} 4=7.8-17.29 \mathrm{pmol} / \mathrm{L}, \mathrm{fT} 3=3.38-6.45,{ }^{11} \mathrm{fT} 4=10.3-$ $21.9 \mathrm{pmol} / \mathrm{L}$ and fT3 $=3.1-7-7 \mathrm{pmol} / \mathrm{L},{ }^{12} \mathrm{fT} 33.2-6.5 \mathrm{pmol} / \mathrm{L}$, fT4 $12-23 \mathrm{pmol} / \mathrm{L},{ }^{13}$ ESS- described as serum fT3 $<2.3 \mathrm{pg} / \mathrm{mL}$ NR, not reported 
with severe COVID-19 [8]. A second study found a close relationship between suppressed TSH and high mortality rate and longer hospitalization suggesting that thyrotoxicosis may be clinically relevant in COVID-19 patients [10]. Conversely, another study demonstrated that euthyroid sick syndrome (ESS) characterized by a decreased level of thyroid hormones without an increased secretion of TSH was not associated to increased mortality in patients with COVID19 [13].

\section{Discussion}

Overall, beyond the study of Chen et al. [7], in $44 \%$ to $94 \%$ of the COVID-19 patients assessed in the included studies, TSH levels were in the normal range. The prevalence of thyroid dysfunction, defined as abnormal thyroid function tests (TSH and/or thyroid hormones), largely varies among available studies and can be mild to moderate. In particular, the most frequent reported abnormality is low TSH levels reported in $15 \%$ up to $56 \%$ of patients with COVID-19 in association with low or normal to high fT3 or fT4 whereas high TSH levels were reported only in up to $8 \%$ of patients with COVID-19, respectively [7-13]. In three studies, the prevalence of thyroid dysfunction in COVID-19 patients was found significantly higher compared to controls group (including mainly healthy controls but also non-COVID-19 pneumonia patients) [7, 9, 12]. In particular, the mean TSH values were significantly lower in COVID-19 patients compared to control groups $[7,9,12]$. Four studies reported a correlation between thyroid dysfunction and clinical severity of COVID-19, in particular the degree of the decrease in TSH levels and fT3 levels was positively correlated with the severity of the disease $[7,8,11,13]$. In the Chen et al. [7] study, COVID-19 cases were classified clinically as moderate, severe, and critical according to definition of the National Health Committee of China (www.nhc.gov. cn, accessed on November 14, 2020). The degrees of both TSH and total T3 (TT3) decreases were positively correlated with the severity of the disease. On the other hand, total T4 (TT4) levels alone were not correlated with the gravity of disease. In severe and critical COVID-19 situations, lower TSH levels were reported compared to similar gravity situation in non-COVID-19 pneumonia cases [7]. Gao et al. [8] reported that TSH, fT3 levels and fT3/fT4 ratio were significantly lower in patients with severe COVID-19 than in non-severely ill patients. While the median values of TSH were still in the normal range for most of the patients, fT3 levels were lower than the normal range in $5.9 \%$ of the non-severe patients' group compared to $39.4 \%$ in the severe patients' group $(p<0.001)$. No differences were reported for fT4 levels. Similar results were reported also by Lui et al. [11] who underlined that the reduction of fT3 levels associated with systemic inflammation seemed to be related to patient clinical deterioration while TSH or fT4 levels were not related with disease severity. Finally, Zou et al. [13] in a cohort of 149 patients with COVID-19 detected isolated low fT3 levels in $27.5 \%$ of patients; these patients were older with a prevalence in the female sex, and more severe events and symptoms, such as fever and dyspnea. The presence of thyroid dysfunction with low TT3 or fT3 levels, related to de-iodination in reverse T3, with low or normal TSH levels, in the absence of known thyroid diseases, is not uncommon in severe clinical conditions and defined as non-thyroidal illness syndrome (NTI) or ESS. It has been already reported in previous SARS pandemic and in several severe diseases including serious infections, multi-organ failure, chronic renal failure, acute myocardial infarction, or widespread aggressive tumor $[14,15]$. In particular, the low TSH levels and low fT3 levels can be explained by several factors. First, depletion of TSH-positive cells at a pituitary level has been demonstrated by immunostaining in post-mortem studies as well as damage in thyroid follicular cells. This phenomenon can be related to a possible direct effect of the virus on thyroid cells and to the secretion of cytokines involved in inflammatory condition with central suppression of the thyroid axis. Lania et al. [10] in a cohort of 287 COVID-19 patients reported a status of thyrotoxicosis defined as TSH levels below the normal range $(<0.33 \mathrm{mU} / \mathrm{L})$ in $20.2 \%$ of patients and an inverse correlation between serum TSH values and interleukin-6 (IL-6 levels) confirmed at multivariate analysis. In the Gao et al. [8] study, fT3 levels were also negatively correlated with high sensitive C-reactive protein (hsCRP), IL-6, and tumor necrosis factor (TNF) $\alpha$ levels, while TSH levels with hs-CRP and IL-6 in the entire analyzed population independently from the gravity of the disease but not in the non-survivor group. Lui et al. [11] also reported that elevated CRP levels were independently associated with low fT3 levels as well in Zou et al. [13] study in association with higher procalcitonin levels. Some authors consider also an indirect effect on the pituitary-thyroid axis mediated by the corticoids therapy used for COVID-19 treatment interfering with thyroid axis functionality and inhibiting TSH secretion or by low molecular weight heparin for fT4 abnormalities. Gao et al. [8] re-analyzed thyroid dysfunction in their cohort of patients eliminating patients treated with corticoids (11/100), but the results of their study did not differ, relatively to the really small sample size. Finally, in intensive care patients with ESS, a lower mean thyroid weight is normally present because of a reduction in thyroid follicular size associated with depletion of colloid. If the use of thyroxine replacement in these cases does not seem to improve clinical conditions, this syndrome seems to be of poor prognosis in critical patients [16].

About the correlation between thyroid dysfunction and mortality in patients with COVID-19, controversial findings 
were reported in the included studies when this correlation was assessed [8, 10, 13]. The study of Gao et al. [8] evaluated if thyroid hormone levels could predict all-cause mortality in patients with severe and critical COVID-19 syndrome. The authors reported a decreased risk of all-cause mortality for higher fT3 levels. On the other hand, in the multivariate adjusted models, TSH, fT4, and fT3/fT4 ratios were not significantly related with mortality. In the study of Lania et al. [10], in-hospital mortality rate of $21.4 \%$ has been described and passing from almost $20 \%$ in patients with TSH in the normal range to almost $40 \%$ in patients with thyrotoxicosis and TSH levels $<0.1 \mathrm{mU} / \mathrm{L}$. Furthermore, the duration of hospitalization was longer in patients with low TSH levels compared to patients in euthyroidism or hypothyroidism. On the other hand, no significant correlation was found between the presence of ESS and mortality in Zou et al. study [13]. Two studies evaluated TSH levels and thyroid hormone levels after recovery and in both they recovered to baseline at follow-up [7, 9]. The interpretation of low TSH levels should be carefully related to thyroid hormone levels. In the study of Lania et al., thyroid hormones were available only in 73 patients among the 287 included, and 31 among them presented an overt thyrotoxicosis with fT4 levels above the normal range and normal TSH-receptor antibody (TRAb), but no imaging results were available [10]. In Muller et al. study, 25\% $(N=2)$ of patients with low TSH levels, during recovery period presented with hypothyroidism, marked hypoechogenicity, and heterogeneity at ultrasound, while in $75 \%(N=6)$, thyroid function recovered during follow-up but in association with a mild hypoechoic pattern at neck ultrasound and in some of them a reduced uptake at ${ }^{99 \mathrm{~m}} \mathrm{Tc}-$ pertechnetate scintigraphy [12]. In both these studies, thyroid dysfunction was more likely related to thyrotoxicosis sustained by subacute thyroiditis phenomenon in combination with ESS while antibodies (anti-TSHR, anti-Tg and antiTPO) were negative in most cases.

Overall, our systematic review clearly demonstrates that thyroid dysfunction assessed by thyroid function tests may be present in patients with COVID-19. Additionally, there is an agreement in the literature about the prognostic value or clinical impact of thyroid dysfunction in COVID-19 patients and, in particular, the degree of decrease in TSH level was positively correlated with clinical severity of COVID-19. This is in line with a recent meta-analysis where the presence of a thyroid disease was associated with the severity of COVID-19 [17]. However, despite including 2169 individual COVID-19 cases from 8 studies, this meta-analysis has some limitations: it includes a limited number of retrospective studies or case series and a minority of patient (less than $3 \%$ ) were reported to suffer from (not always well defined) thyroid disorders. It should be underlined that our review has several limitations that hampered the achievement of definitive conclusions on the prevalence and significance of thyroid dysfunction in COVID-19 patients. First, a limited number of articles are included and analyzed. Most importantly, a significant heterogeneity was found across the studies and this hampered the calculation of a pooled prevalence of thyroid dysfunction in COVID-19 patients. Lastly, all the included studies were single-center studies with fair quality. Therefore, high-quality prospective multi-centric studies are needed on this topic.

\section{Conclusion}

In conclusion, literature data show that thyroid dysfunction is present in a significant percentage of patients with COVID-19. Although the World Health Organization (WHO) did not recommend systematic thyroid function testing of hospitalized COVID-19 patients, routine assessment of thyroid function in COVID-19 patients in particular requiring high-intensity care should be considered for the frequent co-occurrence of thyrotoxicosis due to a destructive thyroiditis related to SARS-CoV-2 and/or ESS. Even short transient thyrotoxicosis might increase the cardiovascular risk favoring the cardiovascular complications and arrhythmias described in COVID-19 patients and the identification of ESS could be implemented for patient prognostic stratification. In our opinion, assessment of thyroid function should be considered in symptomatic COVID-19 patients, especially those presenting with fever, high viral load and increased inflammatory markers to have a baseline before introducing interfering drugs as corticoids or heparin.

\section{Declaration}

Conflict of interest LG is a member of the Roche Diagnostics advisory board and has received research grants and speaker honoraria from Roche Diagnostics, IBSA, and Sanofi-Genzyme. Other Authors declare no conflicts of interest.

Informed consent Not applicable.

Ethical approval Not applicable.

\section{References}

1. Hossain MF, Hasana S, Mamun AA, Uddin MS, Wahed MII, Sarker S et al (2020) COVID-19 outbreak: pathogenesis, current therapies, and potentials for future management. Front Pharmacol 16(11):563478. https://doi.org/10.3389/fphar.2020.563478

2. Parolin M, Parisotto M, Zanchetta F, Sartorato P, De Menis E (2020) Coronaviruses and endocrine system: a systematic review on evidences and shadows. Endocr Metab Immune Disord Drug Targets. https://doi.org/10.2174/1871530320666200905123332

3. Marazuela M, Giustina A, Puig-Domingo M (2020) Endocrine and metabolic aspects of the COVID-19 pandemic. Rev Endocr 
Metab Disord 21(4):495-507. https://doi.org/10.1007/s1115 4-020-09569-2

4. Rotondi M, Coperchini F, Ricci G, Denegri M, Croce L, Ngnitejeu ST et al (2020) Detection of SARS-COV-2 receptor ACE-2 mRNA in thyroid cells: a clue for COVID-19-related subacute thyroiditis. J Endocrinol Invest 6:1-6. https://doi.org/10.1007/ s40618-020-01436-w

5. Moher D, Liberati A, Tetzlaff J, Altman DG, The PRISMA Group (2009) Preferred reporting items for systematic reviews and metaanalyses: the PRISMA statement. BMJ 2009(339):b2535. https:// doi.org/10.1136/bmj.b2535

6. van Gerwen M, Alsen M, Little C, Barlow J, Naymagon L, Tremblay D et al (2020) Outcomes of patients with hypothyroidism and COVID-19: a retrospective cohort study. Front Endocrinol (Lausanne) 18(11):565. https://doi.org/10.3389/fendo.2020.00565

7. Chen M, Zhou W, Xu W (2020) Thyroid function analysis in 50 patients with COVID-19: a retrospective study. Thyroid. https:// doi.org/10.1089/thy.2020.0363

8. Gao W, Guo W, Guo Y, Shi M, Dong G, Wang G et al (2020) Thyroid hormone concentrations in severely or critically ill patients with COVID-19. J Endocrinol Invest 2:1-10. https://doi. org/10.1007/s40618-020-01460-w

9. Khoo B, Tan T, Clarke SA, Mills EG, Patel B, Modi M et al (2020) Thyroid function before, during and after COVID-19. J Clin Endocrinol Metab 12:dgaa830. https://doi.org/10.1210/clinem/dgaa830

10. Lania A, Sandri MT, Cellini M, Mirani M, Lavezzi E, Mazziotti G (2020) Thyrotoxicosis in patients with COVID-19: the THYRCOV study. Eur J Endocrinol 183(4):381-387. https://doi. org/10.1530/EJE-20-0335

11. Lui DTW, Lee CH, Chow WS, Lee ACH, Tam AR, Fong CHY et al (2020) Thyroid dysfunction in relation to immune profile, disease status and outcome in 191 patients with COVID-19. J Clin Endocrinol Metab 3:dgaa813. https://doi.org/10.1210/cline $\mathrm{m} / \mathrm{dgaa} 813$

12. Muller I, Cannavaro D, Dazzi D, Covelli D, Mantovani G, Muscatello A et al (2020) SARS-CoV-2-related atypical thyroiditis. Lancet Diabetes Endocrinol 8(9):739-741. https://doi.org/10.1016/ S2213-8587(20)30266-7

13. Zou R, Wu C, Zhang S, Wang G, Zhang Q, Yu B et al (2020) Euthyroid sick syndrome in patients with COVID-19. Front Endocrinol (Lausanne) 7(11):566439. https://doi.org/10.3389/fendo .2020 .566439

14. Wei L, Sun S, Xu CH, Zhang J, Xu Y, Zhu H et al (2007) Pathology of the thyroid in severe acute respiratory syndrome. Hum Pathol 38:95-102

15. Fliers E, Bianco AC, Langouche L, Boelen A (2015) Thyroid function in critically ill patients. Lancet Diabet Endocrinol 3:816-825

16. Rothberger GD, Valestra PK, Knight K, Desai AK, Calixte R, Shapiro LE (2019) Low free T 3 is associated with worse outcomes in patients in the ICU requiring invasive mechanical ventilation. J Intensiv Care Med. https://doi.org/10.1177/0885066619890822

17. Hariyanto TI, Kurniawan A (2020) Thyroid disease is associated with severe coronavirus disease 2019 (COVID-19) infection. Diabetes Metab Syndr 14(5):1429-1430. https://doi.org/10.1016/j. dsx.2020.07.044

Publisher's Note Springer Nature remains neutral with regard to jurisdictional claims in published maps and institutional affiliations. 